\title{
Chemical Biology-Based Data-Extraction: Monitoring Kinase Activity inside the Mitochondria
}

Vyas Sharma*

Division of Medicinal Chemistry and Natural Products, The University of North Carolina, USA

\begin{abstract}
To confront the multi-dimensional complexity of biological systems, chemical tools are needed that can extract multi-dimensional data from such systems. Many cellular responses to stimuli are regulated by the activities of protein kinase enzymes, their dynamic movements, and their interactions at various subcellular locations. Multi-dimensional data, that includes the activities of signaling-components, and their intracellular location, can be extracted using fluorescent-sensors. Robust peptide-based fluorescent-sensors of kinase activity, developed by the application of the Deep-Quench approach, have recently been reported. Using these sensors PKA activity was monitored in vitro and inside the Mitochondria.
\end{abstract}

Graphical Abstract: The relative distribution of PKA activities in the Mitochondria.

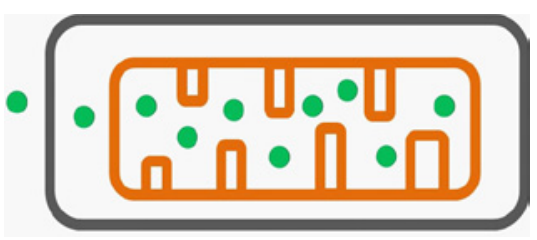

\section{Introduction}

Biological-systems possess immense complexity. Typical biologicalsystems; such as, macromolecules, organelles, cells, organs, organisms, or populations of organisms; consist of more than one biologicalcomponent; and each one of these components can interact with itself, and with other biological-, as well as non-biological components. Furthermore, each one of these components exhibits more than one function; that in turn, depends on multiple factors; which include their structure; their spatial-abundance; and, their assembly and disassembly. Intractability is also experienced with biological-systems, in addition to the factors related to their multi-level and multidimensional complexity, due to another important reason. This reason stated plainly, is that the existence of biological systems, in their present form, is attributed to the forces of evolution and natural-selection that have molded such systems over a period spanning four-billion years. Thus, biological-systems are most appropriately understood as entities that have been engineered; and in many aspects, the task of understanding engineered-entities is an extensive exercise in reverseengineering [1]. In these exercises, design-decisions are deduced from end-products with little or no knowledge about the procedures that went into their production. One typical approach used to understand the construction-and-functioning of an engineered-device, is to extract from this device any, and all, forms of data. The intellectuallandscape of Biology contains numerous data-extraction themes, and new themes continue to emerge fromother disciplines, including the discipline of chemical-biology. One branch of chemical-biology that seeks to gather multi-level and multi-dimensional information from biological-systems is dedicated to the design and application of peptide-based fluorescent-biosensors of kinase activity [2]. This branch of chemical-biology, (located at the intersection of signal-transduction, fluorescence-spectroscopy, and peptide-synthesis), primarily seeks to integrate reductionist- and systems-based approaches within Biology, and to study the biological-components of cell-signaling pathways; the protein kinases,in their natural environments; namely, inside livingcells.

\section{Protein Kinases are Directed to Specific Subcellular Locations during Cell-Signaling}

Most cellular conversations take place on multiple networks that consist of many different cell-signaling cascades; yet, the formation of a majority of these cascades, as well as the flow of information through them, is controlled bythe action of one particular set of enzymes, the protein kinases. These enzymes catalyze the transfer of the $\gamma$-phosphoryl group of ATP to the side chain oxygen atom of an amino acid residue within a protein or peptide substrate. Consequently, when such phosphorylated-products and their downstream signalingcomponents bind a cellular-signal is relayed. Binding-interactions within signaling-networks, also occur between biological receptors and chemical-or environmental-stimuli (that can be non-biological); or, between two or more biological-components; such as, cellular receptors and protein kinases; protein kinases and other protein kinases; protein kinases and anchoring-proteins; and, the phosphorylated-products of protein kinases and various phosphatases; just to name a few. In addition, most cellular-signals are typically initiated at one location within a cell, for example at the plasma-membrane, and then relayed to other locations, for example to the cytoplasm, or to the nucleus. Thus, not only is a specific binding-interaction important in the relay of cellsignals (and in their termination), but the subcellular-location where this interaction occurs, is also important. For example, the significance of spatial- and temporal-regulation in cell-signaling is illustrated [3]

*Corresponding author: Vyas Sharma, Research Assistant Professor, Division of Medicinal Chemistry and Natural Products, The University of North Carolina, Chapel Hill, USA, Tel: (919) 966 1146; E-mail: vsharma@email.unc.edu

Received April 03, 2012; Accepted April 03, 2012; Published April 05, 2012

Citation: Sharma V (2012) Chemical Biology-Based Data-Extraction: Monitoring Kinase Activity inside the Mitochondria. Med chem 2:e102. doi:10.4172/21610444.1000e102

Copyright: ( 2012 Sharma V. This is an open-access article distributed under the terms of the Creative Commons Attribution License, which permits unrestricted use, distribution, and reproduction in any medium, provided the original author and source are credited. 
in PC12 cells, in which, one form of chemical-stimulation activated a protein kinase, in the nucleus in a sustained-manner, and induced celldifferentiation; while, another form of chemical-stimulation activated the same protein kinase, however, this time in the cytoplasm, and in a transient-manner, to induce, a completely different response; namely, cell-proliferation.

The signal-carrying- and the signal-relaying-components of PKA based cell-signaling networks are also dynamic, and thus they capable of direction to different subcellular locations. PKA-based signalingcascades are classically activated when an extracellular-ligand binds to G-protein-coupled receptors on the plasma membrane. Ligandbinding stimulates adenylate cyclase enzymes to generate cAMP. Upon binding to cAMP, inactive PKA holoenzyme, releases its active catalytic subunit; and subsequently, this active PKA subunit distributes to various subcellular locations including those containing AKAPs (or A Kinase anchoring proteins) [4]. Thus, dynamic-movements possibly regulate the timing and the intensity of PKA based cell-signals at various subcellular locations, and these movements, in turn, regulate the organelles, and ultimately, a cell's response to stimuli.

\section{Fluorescent-Sensors Generate Multi-Dimensional Data}

The importance of the dynamic-nature of cell-signaling networks and their components is well recognized; yet, the importance of extracting dynamic-information from cellular-systems is only recently being appreciated. Such data-extraction efforts require tools and protocols that are capable of generating multi-dimensional data. Unfortunately, the application of a reductionist-approach to cellsignaling results in the loss of dynamic information; for example, in vitro kinase assays typically have poor spatial- and temporal-resolution. Yet, these assays are also indispensable for studying the kinetic properties of isolated kinases. In vitro assays can provide a wealth of enzymatic information including, substrate-preferences, the rates of catalysis, and the effects that inhibitors, activators, and structural-modifications, have on kinase activity. The most frequently used in vitro assay for determining kinase activity is the radioactive ATP assay, which detects the amountof $32 \mathrm{P}$, or $33 \mathrm{P}$, incorporated into a peptide- or proteinsubstrate. Theoretically, this assay can be used to measure the activity of any kinase; and, along with its universal-applicability, it is also highlysensitive. However, it also has certain disadvantages. Primarily, its format is discontinuous; its readout is one-dimensional (CPMs); its use of radioactivity requires special precautions; and, it is impractical inside living-cells. One major advantage of moving from this radioactivity based assay towards fluorescent-kinase-sensors is that radioactivityuse can be eliminated. Furthermore, not only are fluorescent-sensors capable of detection-levels down the single-molecule scale, they can also be used in both in vitro, and in organelle-based, or cell-based assay formats; and thus, they are capable of generating multi-dimensional information at two or more biological-levels. In addition, fluorescent molecules possess many intrinsic properties that make them more attractive reporters of enzymatic-activity than radioisotopes. These properties include: polarization, FRET, metal-ion enhancement, environmental-sensitivity, excimer-formation, quenching, and the access to an entire spectrum of different emission wavelengths. Such properties also provide an unprecedented level of multi-dimensionality in measurements. In short, fluorescent-sensors can generate sensitive and continuous multi-dimensional information that not only includes time and space, but also includes color [5].

Within modern Biology's intellectual landscape, one perspective of a fluorescence-based multi-dimensional data-extraction theme is provided by the green-fluorescent proteins (GFPs) [6]. These fluorescent proteins, and their many genetically-modified constructs, have formed a bridge between fluorescence-technology, and the discipline of molecular biology. Molecular biology is widely-recognized as one of the central-forces to have advanced Biology from a hypothesisonly driven field, into a modern-science that is now also driven by discovery. Furthermore, due to the contributions of this discipline, the DNA molecule, once considered an intractable sticky-mess, is now one of the easiest biological macromolecules to analyze and manipulate. Hence, by virtue of being genetically-encoded fluorescence-reporters it is no surprise that the GFPs have formed a seamless-conduit between molecular biology and fluorescence-technology. Not only has the synergy of these two fields generated numerous robust and multicolored fluorescent proteins; it has also allowed us to witness the lifestories of many endogenously-expressed proteins (fused with GFPs) on the cellular stage [7]. GFP-based applications, such as geneticallyencoded FRET-based sensors have also reached cell-signaling [8] .Yet, despite GFP's numerous contributions to cell-biology, (and many contributions that are still to come), GFPs do have some disadvantages. First, they are relatively-large proteins; and second, prior to their use, cells need to be genetically-modified. Thus, as fluorescent-reporters, GFPs are not very "silent". The very act of tagging a GFP molecule onto another protein is likely to perturb the natural behavior of that protein, and subsequently, the natural behavior of the cell. Furthermore, it is also likely that data generated by GFP-based approaches, or other approaches requiring genetic modifications, will be tinted by the biological-version of the observer-effect [9]; whereby, the very act of observation affects the study. Although, the observer-effect cannot be overcome completely, it certainly can be reduced; for example, if the system under-study was perturbed less-drastically. If we compare GFP-based FRET sensors of kinase activity, that only provide modest changes $(<50 \%)$; with, peptide-based fluorescent-sensors that can provide rather large, and robust changes (between 10-1000 fold); then, the peptide-based fluorescent-sensors are anticipated to perform their functions more silently than GFPs (as long as these modified- peptide sensors are able find their way into cells). Furthermore, peptide-based fluorescent-sensors enjoy other advantages over GFPs because they are products of organic synthesis; and thus, structural modifications, including unnatural substituents, can be incorporated into these sensors to generate a level of structural-diversity, that is not yet accessible to molecular biology.

\section{Robust In vitro Sensors of PKA Activity can be obtained by the Deep-Quench Approach}

Peptide-based fluorescent-sensors can be generated via solid-phase peptide synthesis. In one adaptation of a highly successful approach, [10] peptide libraries are designed from kinase consensus-sequences, and fluorescent-molecules appended at different positions. These libraries are subsequently screened for changes in fluorescence upon phosphorylation. Typically, changes in fluorescence are not due to the generation of new fluorescence, but rather due to changes within the intrinsic properties of the appended fluorescent-molecules. Librarymembers that are able to fluorescently respond to their phosphorylation status are selected as biosensors. In the Deep-Quench variation to this approach, a peptide-library, a dye library, and a phosphopeptide binding domain; are employed [11,12]. In the absence of phosphorylation, the dye quenches the peptide's fluorescence; however, upon phosphorylation, the phospho-peptide binding domain binds and sequesters the product from the dye, thereby relieving the quenching, and restoring the fluorescence. Using this approach, pyrene-based (em $\sim 380 \mathrm{~nm}$ ) and coumarin-based (em $\sim 430 \mathrm{~nm}$ ) peptide-libraries were screened for PKA-dependent responses, and robust in vitro sensors of PKA activity ( 20-150 fold enhancements) were obtained $[11,12]$. 
Citation: Sharma V (2012) Chemical Biology-Based Data-Extraction: Monitoring Kinase Activity inside the Mitochondria. Med chem 2:e102. doi:10.4172/2161-0444.1000e102

Page 3 of 3

\section{Deep-Quench Sensors Report PKA Activity inside the Mitochondria}

The mitochondrion, a cellular organelle, once considered as freefloating rigid energy-generator is now recognized as dynamic cellsignaling-platform that is known to undergo drastic morphological changes; including fission and fusion [13]. Interest has increased in the subcellular activities of PKA, inside the mitochondria, due to the appreciation of this organelle's role as a cell-signaling hub; [14] as well as the recognition of PKA's role within this organelle during its fission [15]. Deep-Quench peptide-based in vitro fluorescentsensors were capable of detecting PKA activity in isolated bovine heart mitochondria, [12] and these sensors revealed that the relative matrix/ intermembrane space/outer membrane distribution of PKA is 85/6/9. Our ability to estimate the quantities of active PKA using fluorescent sensors, within various compartments of this organelle, is only the first step to understanding PKA-mediated organelle-based, and cell-based, signaling-systems in real-time.

\section{Conclusions and Perspectives}

The peptide based fluorescent-sensors reported thus far have found fewer applications inside the cell, than outside. This is partly because such sensors are not cell-permeable; and they have to be coaxed into the cell. Furthermore, progress in evenly distributing these probes in specific subcellular locations; and, modifications to the cell, or to the sensors, that render these probes resistant to proteolysis, and phosphatases, are still ongoing. Yet, the ability of these sensors to detect PKA activity inside the mitochondria is encouraging; as one of the goals of this branch of chemical-biology is to create tools, with which we can one day silently tap-into a living-cell, and listen-in on, not only its intracellular chit-chat, but also on its more profound conversations about matters of cell-division and apoptosis.

\section{Acknowledgements}

The author thanks Professor David Lawrence for his support.

\section{References}

1. Wooley JC, Lin HS (2005) Catalyzing Inquiry at the Interface of Computing and Biology. National Academies Press, Washington (DC), USA.

2. Sharma V, Wang Q, Lawrence DS (2008) Peptide-based fluorescent sensors of protein kinase activity: design and applications. Biochim Biophys Acta 1784: 94-99.

3. Marshall CJ (1995) Specificity of receptor tyrosine kinase signaling: transient versus sustained extracellular signal-regulated kinase activation. Cell 80: 179185 .

4. Feliciello A, Gottesman ME, Avvedimento EV (2001) The biological functions of A-kinase anchor proteins. J Mol Biol 308: 99-114.

5. Wang Q, Zimmerman El, Toutchkine A, Martin TD, Graves LM, et al. (2010) Multicolor monitoring of dysregulated protein kinases in chronic myelogenous leukemia. ACS Chem Biol 5: 887-895.

6. Tsien RY (2009) Constructing and exploiting the fluorescent protein paintbox (Nobel Lecture). Angew Chem Int Ed Engl 48: 5612-5626.

7. Kaiser $J(2008)$ Systems biology. Cast of 1000 proteins shines in movies of cancer cells. Science 322: 1176-1177.

8. Ni Q, Titov DV, Zhang J (2006) Analyzing protein kinase dynamics in living cells with FRET reporters. Methods 40: 279-286.

9. Sharma V, Lawrence DS (2009) Über-Responsive Peptide-Based Sensors of Signaling Proteins. Angew Chem Int Ed Engl 48: 7290-7292.

10. Lawrence DS (2005) Signaling protein inhibitors via the combinatorial modification of peptide scaffolds. Biochim Biophys Acta 1754: 50-57.

11. Sharma V, Agnes RA, Lawrence DS (2007) Deep Quench: An Expanded Dynamic Range for Protein Kinase Sensors. J Am Chem Soc 129: 2742-2743.

12. Agnes RS, Jernigan F, Shell JR, Sharma V, Lawrence DS (2010) Suborganelle Sensing of Mitochondrial cAMP-Dependent Protein Kinase Activity. J Am Chem Soc132: 6075-6080.

13. Braschi E, McBride HM (2010) Mitochondria and the culture of the Borg BioEssays 32: 958-966.

14. Thomson M (2002) Evidence of undiscovered cell regulatory mechanisms: phosphoproteins and protein kinases in mitochondria. Cell Mol Life Sci 59: 213219

15. Chang CR, Blackstone C (2007) Cyclic AMP-dependent protein kinase phosphorylation of Drp1 regulates its GTPase activity and mitochondrial morphology. J Biol Chem 282: 21583-21587. 